\title{
Geographic and facility variation in initial use of non-tunneled catheters for incident maintenance hemodialysis patients
}

Edward G. Clark ${ }^{1, *^{*}}$, Ayub Akbari', Brett Hiebert², Swapnil Hiremath', Paul Komenda ${ }^{3}$, Charmaine E. Lok ${ }^{4}$, Louise M. Moist ${ }^{5}$, Michael E. Schachter ${ }^{6}$, Navdeep Tangri ${ }^{7}$ and Manish M. Sood ${ }^{1}$

\begin{abstract}
Background: Non-tunneled (temporary) hemodialysis catheters (NTHCs) are the least-optimal initial vascular access for incident maintenance hemodialysis patients yet little is known about factors associated with NTHC use in this context. We sought to determine factors associated with NTHC use and examine regional and facility-level variation in NTHC use for incident maintenance hemodialysis patients.

Methods: We analyzed registry data collected between January 2001 and December 2010 from 61 dialysis facilities within 12 geographic regions in Canada. Multi-level models and intra-class correlation coefficients were used to evaluate variation in NTHC use as initial hemodialysis access across facilities and geographic regions. Facility and patient characteristics associated with the lowest and highest quartiles of NTHC use were compared.

Results: During the study period, 21,052 patients initiated maintenance hemodialysis using a central venous catheter (CVC). This included 10,183 patients (48.3\%) in whom the initial CVC was a NTHC, as opposed to a tunneled CVC. Crude variation in NTHC use across facilities ranged from 3.7 to $99.4 \%$ and across geographic regions from 32.4 to $85.1 \%$. In an adjusted multi-level logistic regression model, the proportion of total variation in NTHC use explained by facility-level and regional variation was $40.0 \%$ and $34.1 \%$, respectively. Similar results were observed for the subgroup of patients who received greater than 12 months of pre-dialysis nephrology care. Patient-level factors associated with increased NTHC use were male gender, history of angina, pulmonary edema, COPD, hypertension, increasing distance from dialysis facility, higher serum phosphate, lower serum albumin and later calendar year.
\end{abstract}

Conclusions: There is wide variation in NTHC use as initial vascular access for incident maintenance hemodialysis patients across facilities and geographic regions in Canada. Identifying modifiable factors that explain this variation could facilitate a reduction of NTHC use in favor of more optimal initial vascular access.

Keywords: Vascular access, Hemodialysis, Temporary hemodialysis catheters, Epidemiology, Practice variation

\footnotetext{
* Correspondence: edclark@toh.on.ca

'Division of Nephrology, Department of Medicine, The Ottawa Hospital and Kidney Research Centre, Ottawa Hospital Research Institute, University of Ottawa, Ottawa, ON, Canada

${ }^{8}$ The Ottawa Hospital - Riverside Campus, 1967 Riverside Drive, Ottawa, ON K1H 7 W9, Canada

Full list of author information is available at the end of the article
} International License (http://creativecommons.org/licenses/by/4.0/), which permits unrestricted use, distribution, and reproduction in any medium, provided you give appropriate credit to the original author(s) and the source, provide a link to the Creative Commons license, and indicate if changes were made. The Creative Commons Public Domain Dedication waiver (http://creativecommons.org/publicdomain/zero/1.0/) applies to the data made available in this article, unless otherwise stated. 


\section{Background}

Non-tunneled (temporary) hemodialysis catheters (NTHCs) are the preferred initial vascular access for patients with acute kidney injury (AKI) [1]. However, for patients initiating chronic hemodialysis, NTHCs are the least optimal initial vascular access [2-4]. In this situation, the insertion of a NTHC is an additional procedure, with risks of serious complications [3], for patients who will subsequently require another procedure to establish permanent vascular access anyway. Similar to what has been well described for tunneled catheters [3], likely related to consequent central venous stenosis [1], there is some evidence to suggest that initial use of a NTHC is associated with later vascular access complications such as arteriovenous fistula (AVF) thrombosis [5].

Despite efforts aimed at improving access to and provision of pre-dialysis nephrology care to minimize the use of central venous catheters (CVCs) (tunneled CVCs and NTHCs) [6], CVCs remain the initial vascular access for up to $80 \%$ of chronic hemodialysis patients in Canada [7]. Little is known about the use of NTHCs as previous studies have not distinguished between tunneled CVCs and NTHCs [8-10].

Regional variation in dialysis practices, either at the facility or geographic level, has been well documented in Canada and other jurisdictions [11-20]. Interventions targeting a reduction in measured practice variation have translated into improvements in patient care $[18,19]$. As such, quantitating practice variation for key dialysis performance metrics, such as vascular access, could facilitate the development of programs and policies to improve care. To our knowledge, no studies have sought to examine practice variation in NTHC use in patients starting maintenance hemodialysis. In this study, we set out to measure facility and geographic variation for the initial use of NTHCs and determine the factors among patients and facilities that are associated with greater NTHC use. Identification of these factors and subsequently classifying them as either modifiable or non-modifiable would potentially help in planning and implementation of process measures designed to reduce variation.

\section{Methods}

\section{Population and data sources}

All adult ( $>18$ years old) patients who started maintenance hemodialysis with a CVC from January 2001 to December 2010, captured in the Canadian Organ Replacement Registry (CORR), were included in our study. CORR is a validated registry that includes information (patient demographics, comorbidities, modality of RRT, transplantation, vascular access type and survival statistics) on all end stage kidney disease (ESKD) patients in Canada (excluding the province of Québec) who start on dialysis
$[21,22]$. Ethics approval was obtained from the Ottawa Health Science Network Research Ethics Board. Use of CORR was approved by the Canadian Institute for Health Information (CIHI).

\section{Definitions}

Vascular access was defined as the vascular access used for the first hemodialysis treatment. Patients using an arteriovenous fistula (AVF) or graft (AVG) were excluded, as were patients with a co-existing AVF or AVG and CVC. CVCs are prospectively coded within CORR as either being NTHCs or tunneled CVCs. Data regarding the side (right/left) and anatomical site of catheter placement (i.e. internal jugular, subclavian or femoral) is unavailable in the registry. First visit date with a nephrologist was used to estimate the length of pre-dialysis nephrology care and categorized as either greater or less than 1 year prior to first dialysis. Distance to facility was defined as less than 50, 50 to 150 and greater than 150 kilometers, using previously published methods [23]. Individual patients and dialysis facilities were de-identified for analytic purposes. The presence of co-morbidities (angina, chronic obstructive pulmonary disease, diabetes, malignancy, serious illness, hypertension, lung disease, coronary artery bypass grafting, pulmonary edema, peripheral vascular disease, stroke, cigarette smoker, acute coronary syndrome) and laboratory values immediately prior to dialysis initiation (hemoglobin, albumin, phosphate) are captured within CORR.

Since dialysis care may vary across facilities, facility level variables were created based on clinical relevance, known association with outcomes, and quality of care indicators [24-27]. Facility-level variables included whether or not the facility offered kidney transplantation or peritoneal dialysis, mean hemoglobin and phosphate of a facility's patients at dialysis initiation, average distance in kilometers between a facility's patients' primary residences and nearest dialysis facility, center size and average estimated glomerular filtration rate (eGFR) at dialysis initiation. Information on patients geographic regions was included and categorized into 12 regions as Atlantic, Northern Ontario, Greater Toronto, Eastern Ontario, Western Ontario, Manitoba, Saskatchewan, Northern Alberta, Southern Alberta, Eastern British Columbia, Vancouver and Other British Columbia regions. Facilities with 10 or less patients during the entire study period were excluded. Multiple imputation was employed for missing values [28].

\section{Statistical analysis}

Patient and facility characteristics were compared between patients who were started on dialysis with a NTHC or tunneled CVC. Continuous variables of interest were summarized using the mean with standard deviation. 
Differences in characteristics were determined by the Student's $t$-test for continuous variables and the chi-square for dichotomous variables.

Facility and geographic variation were examined using a three level, multilevel model and to assess predictors associated with a NTHC. Models were adjusted for factors thought to potentially influence decisions to initiate dialysis including facility level factors (transplantation facility, peritoneal dialysis facility, average hemoglobin and phosphate, average distance a patient resided from the nearest dialysis facility and number of HD patients treated at the facility, average eGFR at dialysis initiation), patient case mix (age, sex, body mass index, race, co-morbidities, distance to facility, serum phosphate, albumin and hemoglobin, eGFR at dialysis initiation) and calendar year. Facility and geographic variation were determined by the intra-class correlation coefficient (ICC) [29]. The intra-class correlation coefficients were calculated by dividing the variance estimate at each level by the total model variance. In our study the intra-class correlation coefficient determines the proportion of explained variation in the use of a NTHC at dialysis initiation that is due to being a member of a particular group such as patient, facility and geographic region and is reported as a percentage. Facility-level variables were centered for the facility-averages [30]. The $\mathrm{R}^{2}$ used to determine the percentage of variation explained at each level for the full and reduced models was determined by the Raudenbush and Byrk method, whereas the $\mathrm{R}^{2}$ for the total model was determined by the Snijder and Bosker method [31, 32]. We employed the SAS GLIMMIX procedure (SAS 9.2) using a logit link and employing latent variable approach at the patient level [30]. In this model the intraclass correlation coefficients were calculated for the facility and geographic regions by assuming a patientlevel variance of $\pi^{2} / 3[33,34]$. To interrogate the patient-level variance assumption of $\pi^{2} / 3$, the ICC was further calculated using a probit link assuming a patient level variance of 1 . We calculated the odds ratios for initiation with a NTHC by geographic region in a separate 2-level multilevel logistic model adjusted for patient case mix and facility-level factors listed previously. As it has been established that late nephrology referral is associated with significantly greater CVC use [35], we conducted an additional analysis limiting our cohort only to patients with pre-dialysis care $>365$ days (i.e. excluding patients with shorter duration of pre-dialysis care).

Lastly, we categorized the facilities into quartiles of NTHC use and compared patient and facility characteristics associated with the highest and lowest quartiles for NTHC use. Analyses were performed using SAS version 9.2 (SAS Inc, Cary NC).

\section{Results}

During the study period, 21,052 patients began chronic dialysis with a CVC, of which 10,183 (48.3\%) were NTHCs. Table 1 details baseline patient, facility and geographic characteristics of the study cohort according whether initial vascular access was a NTHC or a tunneled permanent HD catheter. Patients with a NTHC were less likely to be Caucasian, have shorter pre-dialysis care, have a history of vascular disease and pulmonary edema, have more laboratory abnormalities and reside further from a dialysis facility. Facilities characteristics associated with NTHC use included less likely to be a peritoneal dialysis or transplantation facility and less incident patients.

Crude variation in NTHC use across 61 facilities ranged from 3.7 to $99.4 \%$ and across geographic regions from 32.4 to $85.1 \%$. Fig. 1 shows NTHC use as a percentage of crude CVC use (NTHCs and tunneled HD catheters) across all geographic regions.

Table 2 reports the unadjusted and adjusted attributable variation at the facility and geographic-level for initiation of hemodialysis with a NTHC. In the multi-level logistic regression model, the explained variation at the facility level was $43.9 \%$ unadjusted and $40.0 \%$ adjusted $(p<0.0001)$. Explained variation at the regional levels was $21.8 \%$ and $34.1 \%(p=0.1)$, respectively. Similar results were observed for the subgroup of patients who received $>1$ year of pre-dialysis nephrology care with explained variation of $46 \%$ and $38.1 \%$ at the facility and regional levels, respectively (data not shown). Fig. 2 shows the adjusted odds ratios for initial NTHC use across Canada in which adjusted odds ratios ranged from 0.59 (95\% CI, 0.49-0.71) in the Atlantic region to 9.26 (95\% CI, 6.81-12.58) in BC (Other) with Saskatchewan as the reference group.

Patient and facility characteristics for those facilities within the highest and lowest quartiles of NTHC use at dialysis initiation are compared in Table 3 . In general, patients treated at facilities with the lowest quartile of NTHC use were younger, less likely to be Caucasian, had longer pre-dialysis care and lower eGFR at dialysis initiation. Conversely, patients at facilities in the highest quartile of NTHC use were more likely to have a history of pulmonary edema, stroke, cigarette smoking, PVD, serious illness, reside rurally and less likely to have diabetes, angina or ESRD due to diabetes. Facilities in the top quartile of NTHC use had fewer incident patients, and were less likely to be a transplant or peritoneal dialysis facility.

The extent to which patient and facility level variables were associated with incident NTHC use within the fully adjusted model are reported in Table 4. Lower number of incident patients per facility, male sex, a history of angina, pulmonary edema, lung disease, residing a further distance from a dialysis facility, higher eGFR at dialysis 
Table 1 Baseline patient, facility and geographic characteristics of the study cohort according to individuals who initiated dialysis with non-tunneled temporary vs. tunneled hemodialysis catheters

\begin{tabular}{|c|c|c|c|}
\hline \multicolumn{4}{|l|}{ Characteristic } \\
\hline & NTHC & tCVC & pvalue \\
\hline $\mathrm{N}$ & $10,162(48.3)$ & $10,890(51.7)$ & \\
\hline \multicolumn{4}{|l|}{ Patient Characteristics: } \\
\hline Age $( \pm S D)$ & $64.6 \pm 15.5$ & $64.6 \pm 16.2$ & 0.705 \\
\hline Sex $\%$ male & 59.4 & 57.4 & 0.004 \\
\hline $\mathrm{BMI}( \pm \mathrm{SD})$ & $27.3 \pm 6.6$ & $27.5 \pm 6.7$ & 0.052 \\
\hline Race (\%) & & & $<0.001$ \\
\hline Caucasian & 70.9 & 74.2 & \\
\hline East Asian & 5.9 & 5.1 & \\
\hline Aboriginal & 7.8 & 6.1 & \\
\hline South Asian & 2.5 & 3.8 & \\
\hline Black & 4.2 & 3.9 & \\
\hline Other & 6.2 & 3.4 & \\
\hline Unknown & 2.5 & 3.6 & \\
\hline Pre-dialysis care $>90$ days $\%$ & 53.2 & 64.3 & $<0.001$ \\
\hline Pre-dialysis care $>365$ days $\%$ & 34.2 & 45.6 & \\
\hline \multicolumn{4}{|l|}{ Co-morbidities: \% } \\
\hline Angina & 27.2 & 23.9 & $<0.001$ \\
\hline Acute coronary syndrome & 26.5 & 24.8 & 0.003 \\
\hline Pulmonary edema & 34.1 & 29.4 & $<0.001$ \\
\hline Diabetes mellitus & 46.7 & 49.3 & $<0.001$ \\
\hline Stroke & 16.9 & 16.5 & 0.437 \\
\hline Peripheral vascular disease & 23.3 & 21.6 & 0.003 \\
\hline Malignancy & 15.1 & 15.3 & 0.788 \\
\hline Lung disease & 14.9 & 13.4 & 0.002 \\
\hline Any hypertension medication(s) & 81.3 & 85.6 & $<0.001$ \\
\hline Current smoker & 16.9 & 15.7 & 0.034 \\
\hline CABG & 16.4 & 16.4 & 0.970 \\
\hline Serious IIIness & 16.0 & 16.2 & 0.708 \\
\hline Cause of ESRD \% & & & $<0.001$ \\
\hline Hypertension & 23.6 & 21.4 & \\
\hline Diabetes mellitus & 33.8 & 38.2 & \\
\hline Glomerulonephritis & 12.7 & 14.3 & \\
\hline Obstruction & 2.7 & 2.8 & \\
\hline Interstitial & 2.8 & 2.6 & \\
\hline Polycystic kidney disease & 1.8 & 2.8 & \\
\hline Other & 13.1 & 8.6 & \\
\hline Unknown & 9.5 & 9.2 & \\
\hline eGFR at dialysis initiation ( \pm SD) & $9.6 \pm 4.7$ & $9.6 \pm 4.4$ & 0.435 \\
\hline Hemoglobin g/L (mean $\pm S D)$ & $97.5 \pm 17.7$ & $98.9 \pm 16.9$ & $<0.001$ \\
\hline Phosphatemmol/L (mean \pm SD) & $2.0 \pm 0.8$ & $2.0 \pm 0.7$ & $<0.001$ \\
\hline Albumin g/L (mean $\pm S D)$ & $30.3 \pm 0.9$ & $30.7 \pm 0.9$ & $<0.001$ \\
\hline Distance from facility \% & & & $<0.001$ \\
\hline
\end{tabular}


Table 1 Baseline patient, facility and geographic characteristics of the study cohort according to individuals who initiated dialysis with non-tunneled temporary vs. tunneled hemodialysis catheters (Continued)

\begin{tabular}{|c|c|c|c|}
\hline$<50 \mathrm{~km}$ & 73.8 & 76.7 & \\
\hline $50-150$ & 16.2 & 15.4 & \\
\hline$>150$ & 10.0 & 7.9 & \\
\hline \multicolumn{4}{|l|}{ Facility Characteristics* $(N=61)$} \\
\hline Mean number of incident patients (per year) & $51.3 \pm 34.4$ & $60.0 \pm 42.4$ & $<0.0001$ \\
\hline Transplant facility \% & 47.7 & 52.3 & $<0.0001$ \\
\hline Peritoneal dialysis facility \% & 45.9 & 54.1 & $<0.0001$ \\
\hline Mean eGFR at dialysis initiation $( \pm S D)$ & $9.7 \pm 1.1$ & $9.6 \pm 1.2$ & 0.001 \\
\hline Mean Hemoglobin g/L ( \pm SD) & $102.0 \pm 3.5$ & $102.0 \pm 3.4$ & 0.256 \\
\hline Mean Phosphate $\mathrm{mmol} / \mathrm{L}( \pm \mathrm{SD})$ & $1.9 \pm 0.1$ & $1.9 \pm 0.1$ & 0.002 \\
\hline Mean distance from facility $\mathrm{km}( \pm \mathrm{SD})$ & $56.3 \pm 40.7$ & $50.0 \pm 39.6$ & $<0.0001$ \\
\hline
\end{tabular}

$N$ Number, $S D$ standard deviation, $B M I$ body mass index, IQR intra-quartile range, CABG coronary artery bypass graft, $E S R D$ end-stage renal disease, $g / L$ grams per liter, $\mathrm{mmol} / \mathrm{L}$ millimole per liter, AVF arteriovenous fistula, eGFR estimated glomerular filtration rate in $\mathrm{ml} / \mathrm{min} / 1.73 \mathrm{~m}^{2}$ by the 4 variable MDRD equation, $\mathrm{km}$ kilometer

initiation, and higher serum phosphate were independently associated with NTHC use.

\section{Discussion}

This Canadian cohort study of over 20,000 hemodialysis patients who started dialysis between 2001 and 2010 is the first to describe patient and facility level factors associated with starting chronic hemodialysis using a NTHC. The most striking finding is the extent to which initial NTHC use varies across facilities and regions. After adjustment for patient-level factors (case-mix) and facility-level quality indicators, otherwise unspecified center-level factors accounted for $40 \%$ of total variation and regional-level factors accounted for an additional $34 \%$. This is much higher than the extent to which center-level and regionallevel factors explain variation for other dialysis practices in Canada such as eGFR at time of dialysis initiation (3.1 \% and $0.0 \%$, respectively) [17] and initial use of peritoneal dialysis ( $9.3 \%$ and $3.4 \%$, respectively) [36]. For further comparison, in studies of 173 dialysis facilities in the United States, after adjusting for case-mix, facility variation accounted for only $7.1 \%$ of the total variation with respect to AVF use [18] and $6.7 \%$ of the total variation with respect to dialysis adequacy [19].

Numerous factors may account for facility and regional variation in the use of NTHCs relative to tunneled CVCs. The likelihood of 'suboptimal' initiation of chronic dialysis, defined as having a CVC (either a tunneled catheter or NTHC) as initial vascular access maybe increased by systemic and resource limitations that affect access to timely AVF and AVG creation [8, 37, 38]. Similar considerations likely account for some of the variation observed for initial

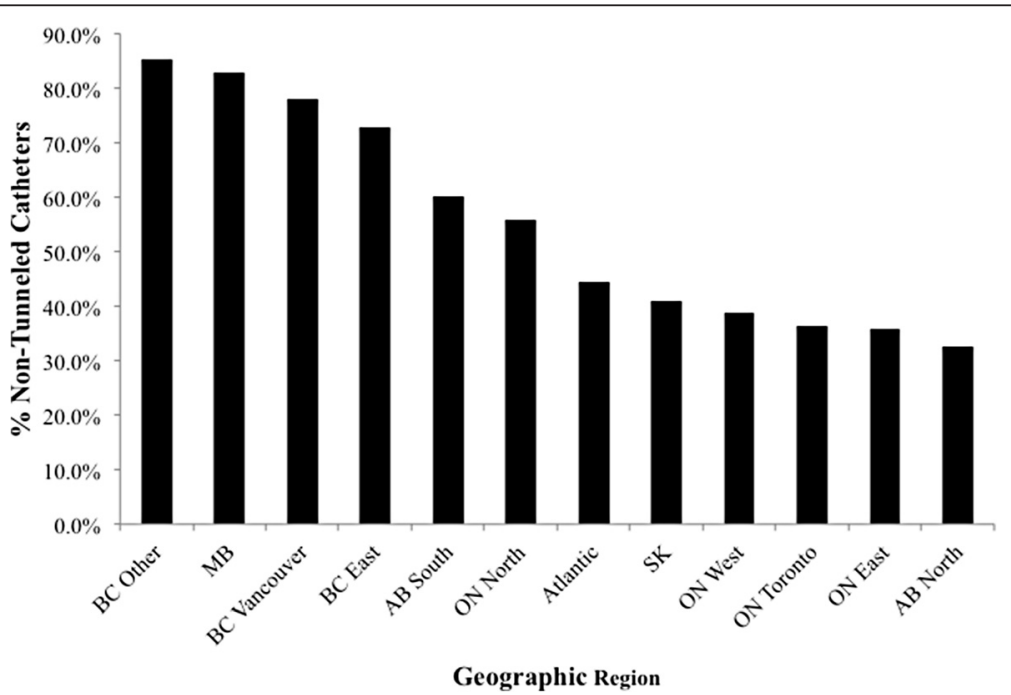

Fig. 1 NTHC use as a percentage of crude CVC use at dialysis initiation, by geographic region. AB, Alberta; BC, British Columbia; MB, Manitoba; SK, Saskatchewan; ON, Ontario 
Table 2 Multi-level model analysis of the unadjusted and adjusted variation at the facility and geographic-level for the initiation of dialysis with a non-tunneled temporary hemodialysis catheter

\begin{tabular}{lllll}
\hline & Intra-class correlation (\%) & Variance estimate & Standard error & $P$ value \\
\hline Unadjusted & & & 0.30 & $<0.0001$ \\
Facility & 43.9 & 1.44 & 0.43 & 0.1 \\
Geography & 21.8 & 0.72 & & $<0.0001$ \\
Fully adjusted & & & 0.30 & 0.1 \\
Facility & 40.0 & 1.32 & 0.68 & 0 \\
Geography & 34.1 & 1.12 & & 0 \\
\hline
\end{tabular}

Geographic regions: 13; Facilities: 61; Patients:21,052

Adjusted for patient-level variables: age, sex, co-morbidities (CVA, angina, PVD, MI, Cancer, Pulmonary edema, COPD, DM, HTN, serious illness, CABG), body-mass index, laboratory values, distance from dialysis facility, cause of ESRD, race, eGFR at dialysis initiation and facility-level variables: \% transplant facility, \% peritoneal dialysis facility, and mean facility laboratory values, mean eGFR at dialysis initiation, mean distance from facility, mean facility size

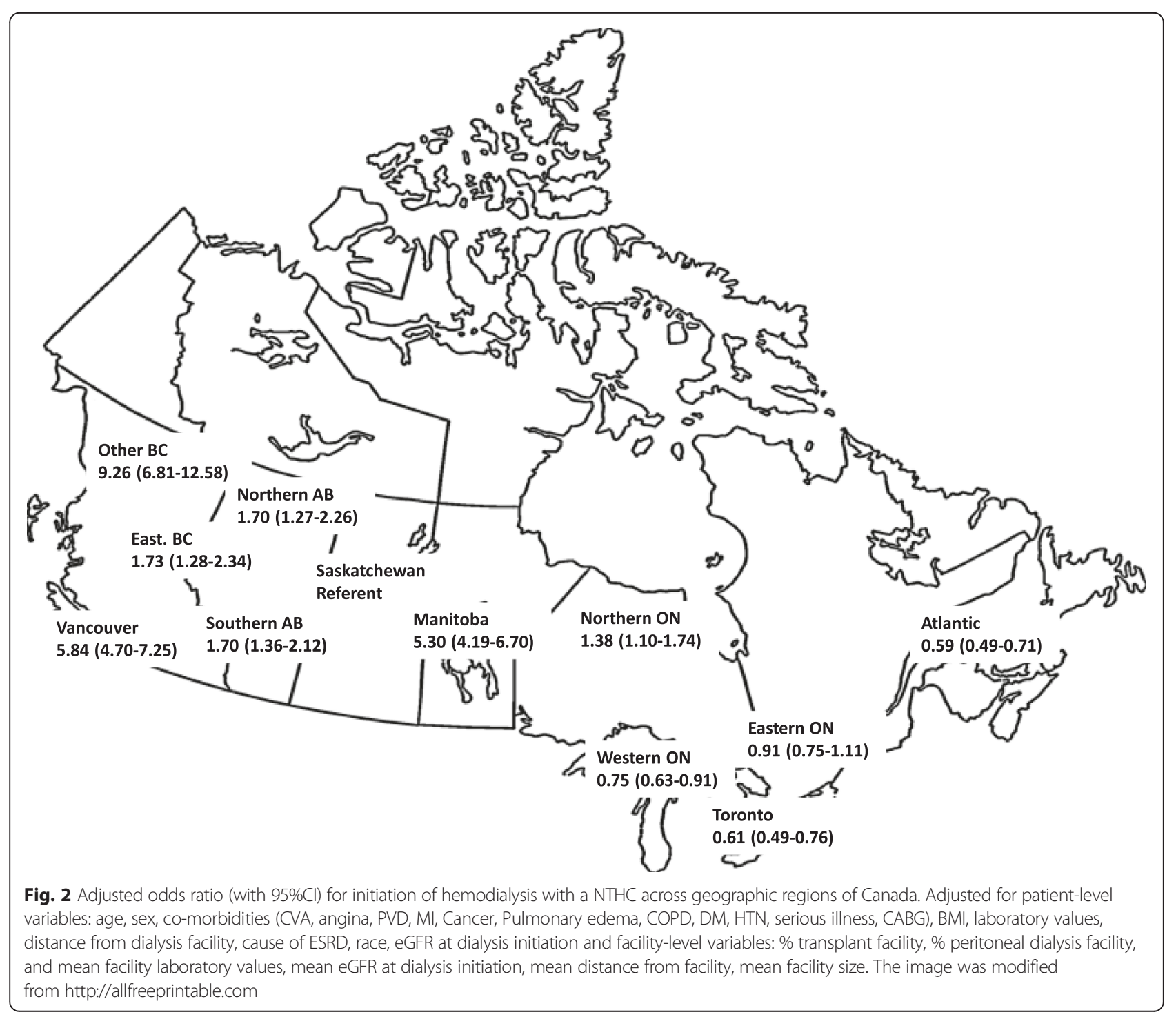


Table 3 Comparative patient and facility characteristics among facilities with the highest and lowest quartile of NTHC use at dialysis initiation

\begin{tabular}{|c|c|c|c|}
\hline \multicolumn{4}{|l|}{ Characteristic } \\
\hline & Lowest quartile NTHC & Highest quartile NTHC & $P$ Value \\
\hline $\bar{N}$ & $5013(51.9)$ & $4651(48.1)$ & \\
\hline$\%$ with temp CVC & 24.8 & 75.2 & \\
\hline Age (mean $\pm S D)$ & $64.0 \pm 15.5$ & $64.8 \pm 15.6$ & 0.01 \\
\hline Sex $\%$ male & 57.9 & 59.2 & 0.395 \\
\hline $\mathrm{BMI}($ mean $\pm \mathrm{SD})$ & $27.4 \pm 6.7$ & $27.6 \pm 6.8$ & 0.123 \\
\hline Race (\%) & & & $<0.001$ \\
\hline Caucasian & 70.9 & 74.2 & \\
\hline East Asian & 5.9 & 5.1 & \\
\hline Aboriginal & 7.8 & 6.1 & \\
\hline South Asian & 2.5 & 3.8 & \\
\hline Black & 4.2 & 3.9 & \\
\hline Other & 6.2 & 3.4 & \\
\hline Unknown & 2.5 & 3.6 & \\
\hline Pre-dialysis care $>90$ days $\%$ & 61.1 & 56.8 & $<0.0001$ \\
\hline Pre-dialysis care $>365$ days $\%$ & 42.3 & 38.1 & $<0.0001$ \\
\hline \multicolumn{4}{|l|}{ Co-morbidities \% } \\
\hline Acute coronary syndrome & 26.3 & 25.1 & 0.177 \\
\hline Pulmonary edema & 33.1 & 29.9 & 0.001 \\
\hline Diabetes mellitus & 48.8 & 44.0 & $<0.001$ \\
\hline Stroke & 14.9 & 16.9 & 0.006 \\
\hline Peripheral vascular disease & 21.2 & 23.0 & 0.035 \\
\hline Malignancy & 14.9 & 15.9 & 0.150 \\
\hline Lung disease & 13.7 & 14.6 & 0.179 \\
\hline Hypertension medications & 85.0 & 77.8 & $<0.001$ \\
\hline Current smoker & 14.8 & 19.3 & $<0.001$ \\
\hline CABG & 13.3 & 14.7 & 0.056 \\
\hline Serious IIIness & 14.5 & 16.3 & 0.015 \\
\hline Angina & 26.4 & 24.4 & 0.03 \\
\hline Cause of ESRD \% & & & $<0.001$ \\
\hline Hypertension & 22.0 & 23.4 & \\
\hline Diabetes mellitus & 38.1 & 28.6 & \\
\hline Glomerulonephritis & 13.6 & 11.3 & \\
\hline Obstruction & 2.8 & 2.1 & \\
\hline Interstitial & 2.8 & 1.8 & \\
\hline Polycystic kidney disease & 2.8 & 2.0 & \\
\hline Other & 8.0 & 20.5 & \\
\hline Unknown & 10.0 & 10.3 & \\
\hline eGFR at dialysis initiation (mean \pm SD) & $9.4 \pm 4.5$ & $9.8 \pm 4.6$ & $<0.0001$ \\
\hline Hemoglobin g/L (mean \pm SD) & $97.9 \pm 17.3$ & $98.2 \pm 17.8$ & 0.341 \\
\hline Phosphate $\mathrm{mmol} / \mathrm{L}($ mean $\pm \mathrm{SD})$ & $2.0 \pm 0.7$ & $2.0 \pm 0.7$ & $<0.0001$ \\
\hline Albumin $\mathrm{g} / \mathrm{L}($ mean $\pm \mathrm{SD})$ & $31.3 \pm 0.9$ & $29.8 \pm 0.9$ & $<0.0001$ \\
\hline Distance from facility \% & & & $<0.0001$ \\
\hline
\end{tabular}


Table 3 Comparative patient and facility characteristics among facilities with the highest and lowest quartile of NTHC use at dialysis initiation (Continued)

\begin{tabular}{llll}
\hline$<50 \mathrm{~km}$ & 75.2 & 72.0 & $<0.0001$ \\
$50-150$ & 18.6 & 16.6 & 11.4 \\
$\quad>150$ & 6.2 & & \\
Facility Characteristics & & $68.6 \pm 35.4$ & $<0.0001$ \\
Mean number of incident patients (per year) & $78.8 \pm 30.3$ & 28.4 & 0.001 \\
Transplant facility \% & 31.6 & 70.5 & $<0.0001$ \\
Peritoneal dialysis facility \% & 99.6 & $9.6 \pm 1.2$ & 0.001 \\
eGFR at dialysis initiation (facility mean \pm SD) & $9.7 \pm 1.1$ & $101.7 \pm 4.2$ & 0.176 \\
Hemoglobin g/L (facility mean \pm SD) & $101.6 \pm 3.5$ & $1.9 \pm 0.1$ & $<0.0001$ \\
Phosphate mmol/L (facility mean \pm SD) & $1.9 \pm 0.2$ & $66.9 \pm 41.5$ & $<0.0001$ \\
Distance from facility km (facility mean \pm SD) & $42.2 \pm 34.7$ &
\end{tabular}

NTHC non-tunelled hemodialysis catheter, N Number, eGFR estimated glomerular filtration rate, SD standard deviation, $B M I$ body mass index, IQR intra-quartile range, $C A B G$ coronary artery bypass graft, ESRD end-stage renal disease, $g / L$ grams per liter, $\mathrm{mmol} / \mathrm{L}$ millimoles per liter, $A V F$ arteriovenous fistula eGFR was estimated glomerular filtration rate in $\mathrm{ml} / \mathrm{min} / 1.73 \mathrm{~m}^{2}$ by the 4 variable MDRD equation, km kilometer

use of NTHCs versus tunneled CVCs across centers and regions. For example, centers that have better access to interventional radiology services or nephrologists and trainees capable of inserting tunneled catheters themselves may be less likely to start patients on dialysis using NTHCs. Some authors have even suggested that the need to provide training opportunities in NTHC insertion to nephrology fellows could result in the "tendency to place a non-tunneled catheter when a tunneled catheter might be more appropriate" [39]. While no jurisdiction in Canada charges patients for dialysis-related services, provider remuneration is determined at the provincial level and could influence practice patterns amongst nephrologists, vascular surgeons, intensivists and interventional radiologists accordingly.

Given that many patients begin dialysis with an NTHC in the context of severe AKI and then may become chronic HD patients in the event of non-recovery, center level variation might be accounted for to some degree by whether or not facilities have acute RRT capability. It is a limitation of this study that details regarding centers' capability to provide acute RRT were not available to be included in the model; nonetheless, given that all regions have secondary or tertiary referral centers that provide RRT for patients with AKI in that region, regional variation is unlikely to be explained on the basis of acute RRT capability. However, regional variation in the incidence of dialysis-requiring AKI has been demonstrated in the United States [40] and similar variation in Canada could at least partially account for regional differences in the propensity for NTHC use.

Notably, similarly pronounced center-level and regional variation was observed for the subset of patients who had received $>1$ year of pre-dialysis nephrology care. It has been shown that suboptimal initiation of dialysis-defined as initiation as an inpatient and/or using a $\mathrm{CVC}$-frequently occurs despite an adequate duration of pre-dialysis nephrology care [19, 38, 41-43] and is associated with increased mortality [42, 44]. We suggest that, in the context of having received an adequate duration of pre-dialysis nephrology care, initiation of chronic dialysis with an NTHC is even worse than 'suboptimal'; it is 'least-optimal'. This is because NTHC insertion in this situation represents an additional procedure carrying risks of serious complications [3] for patients who will require a procedure to establish permanent vascular access anyway. In addition, likely related to consequent central venous stenosis [1], there is some evidence to suggest that initial use of an NTHC is associated with later vascular access complications such as AVF thrombosis [5]. Determining the causes of the unnecessary NTHC use signaled by the wide degree of practice variation could potentially lead to improved pre-dialysis care that diminishes 'least-optimal' dialysis starts.

A particular strength of our study is that data was derived from a large, representative cohort of incident dialysis patients from across Canada (excluding the province of Québec which does not contribute to CORR data). We utilized multi-level models and the intra-class correlation coefficient to quantitate the relative explained variation at individual levels, an analytic strategy that accounts for correlation of observations within clusters. As Canada offers universal public health care, there would be no bias due to unaffordability of health care, vascular access or dialysis services. There was little missing data on vascular access at dialysis initiation $(<7 \%)$ thus our findings are generalizable with limited selection bias.

This study has important limitations related to the use of registry data that did not include information regarding the indication for dialysis initiation including whether or not it was conducted specifically in the setting of AKI. Furthermore, while CORR registry data has been validated 
Table 4 Variables associated with initiation of chronic hemodialysis with a NTHC

\begin{tabular}{|c|c|c|}
\hline Variables & Odds ratio & $95 \%$ Confidence interval \\
\hline \multicolumn{3}{|l|}{ Level 2: Facility variables } \\
\hline Transplant Facility & 0.97 & $0.41-2.30$ \\
\hline Peritoneal Dialysis Facility & 0.89 & $0.33-2.44$ \\
\hline Mean facility Hemoglobin & 0.96 & $0.89-1.05$ \\
\hline Mean facility Phosphate & 0.14 & $0.01-2.30$ \\
\hline Mean facility eGFR at dialysis initiation & 0.92 & $0.70-1.21$ \\
\hline Mean distance from facility & 1.00 & $0.99-1.01$ \\
\hline Number of Patients & 1.00 & $1.01-1.15$ \\
\hline \multicolumn{3}{|l|}{ Level 1: Patient-level variables } \\
\hline Age 59 and Under vs. Age 74+ & 0.96 & $0.88-1.05$ \\
\hline Age 60 to 73 vs. Age $74+$ & 1.05 & $0.97-1.13$ \\
\hline Male & 1.10 & $1.03-1.18$ \\
\hline BMl & 1.00 & $0.99-1.00$ \\
\hline Asian vs. Caucasian & 0.91 & $0.78-1.06$ \\
\hline Black vs. Caucasian & 0.88 & $0.73-1.06$ \\
\hline Indian Subcontinent vs. Caucasian & 0.97 & $0.82-1.15$ \\
\hline Aboriginal vs. Caucasian & 0.93 & $0.80-1.07$ \\
\hline Unknown vs. Caucasian & 0.91 & $0.76-1.08$ \\
\hline Other vs. Caucasian & 0.78 & $0.64-0.94$ \\
\hline Stroke & 0.99 & $0.90-1.08$ \\
\hline Angina & 1.09 & $1.00-1.19$ \\
\hline PVD & 0.97 & $0.89-1.05$ \\
\hline Acute coronary syndrome & 1.06 & $0.97-1.16$ \\
\hline Cancer & 1.03 & $0.94-1.12$ \\
\hline Pulmonary Edema & 1.36 & $1.26-1.46$ \\
\hline Lung disease & 1.11 & $1.01-1.22$ \\
\hline Diabetes & 0.95 & $0.88-1.02$ \\
\hline Hypertension & 0.79 & $0.72-0.86$ \\
\hline Serious IIIness & 1.07 & $0.98-1.17$ \\
\hline CABG & 0.98 & $0.89-1.08$ \\
\hline Distance 50 to 150 vs $<50$ & 1.13 & $1.03-1.25$ \\
\hline Distance $>150$ vs $<50$ & 1.14 & $1.00-1.30$ \\
\hline Phosphate & 1.29 & $1.22-1.36$ \\
\hline Albumin & 0.98 & $0.94-1.02$ \\
\hline Hemoglobin & 0.99 & 0.99-0.99 \\
\hline eGFR at dialysis initiation & 1.02 & $1.01-1.03$ \\
\hline Calendar Year & 0.79 & $0.78-0.80$ \\
\hline
\end{tabular}

to have minimal risk of bias when used for clinical research [22], the coding for 'type of catheter' used for incident dialysis treatment has not specifically been validated. Patients with an AVF/AVG or a co-existent AVF/AVG and $\mathrm{CVC}$ at initial dialysis were excluded and we did not account for subsequent CVC use. A potentially high proportion of patients may experience early AVF/AVG failure and require CVC insertion. Additional limitations include the lack of data pertaining to the anatomic location of catheter insertions (e.g. internal jugular or femoral), where catheters were placed in hospitals (e.g. intensive care unit, ward, dialysis unit, radiology suite), and by which kinds of physicians (e.g. interventional radiologists, nephrologists, intensivists). A final limitation is that, while we attempted 
to account for facility-level complexity of care by assessing if facilities offered transplantation and/or peritoneal dialysis programs, this is not a precise way to distinguish tertiary and quaternary care facilities from others. This is particularly relevant since such centres may also be more likely to provide on-call interventional radiology service and/or acute dialysis therapy for AKI, factors that might affect the type of catheters being used (i.e. NTHC versus tunneled CVC).

\section{Conclusions}

In conclusion, a significant proportion of the variation in initial NTHC use in chronic hemodialysis patients is explained at the facility and regional level. Given that starting dialysis with a NTHC is even less optimal than starting with a tunneled CVC, future studies are needed to determine what underpins facility-level and regionallevel variation: an improved understanding of this variation could lead the way to a reduction in the frequency of 'least-optimal' dialysis starts using NTHCs.

\section{Competing interests}

The authors have no competing interests to declare.

\section{Authors' contributions}

Research idea: MMS; study design: MMS, EGC; data acquisition: MMS, BH; data analysis/interpretation: EGC, AA, SH, PK, CEL, LMM, MES, NT, MMS; statistical analysis: BH. Each author contributed important intellectual content during manuscript drafting or revision. All authors read and approved the final manuscript.

\section{Acknowledgements}

EGC and MMS acknowledge the institutional support for this work provided by the Kidney Research Centre, Ottawa Hospital Research Institute, University of Ottawa, Ottawa, Ontario, Canada.

\section{Author details}

'Division of Nephrology, Department of Medicine, The Ottawa Hospital and Kidney Research Centre, Ottawa Hospital Research Institute, University of Ottawa, Ottawa, ON, Canada. ${ }^{2}$ Cardiac Sciences Program, St Boniface Hospital, Winnipeg, MB, Canada. ${ }^{3}$ Section of Nephrology, Department of Medicine, University of Manitoba, Winnipeg, MB, Canada. ${ }^{4}$ Division of Nephrology, Department of Medicine, Toronto General Hospital and University of Toronto, Toronto, ON, Canada. ${ }^{5}$ Division of Nephrology, Department of Medicine, Schulich School of Medicine and Dentistry, Western University and Kidney Clinical Research Unit, London Health Sciences Centre, London, ON, Canada. ${ }^{6}$ Royal Jubilee Hospital, Victoria, BC, Canada. ${ }^{7}$ Seven Oaks Hospital, Winnipeg, MB, Canada. ${ }^{8}$ The Ottawa Hospital - Riverside Campus, 1967 Riverside Drive, Ottawa, ON K1H 7 W9, Canada.

Received: 5 August 2015 Accepted: 19 February 2016

Published online: 27 February 2016

\section{References}

1. Kidney Disease: Improving Global Outcomes (KDIGO) Acute Kidney Injury Work Group. KDIGO Clinical Practice Guideline for Acute Kidney Injury. Kidney Inter Suppl. 2012;2:1-138.

2. National Kidney Foundation. K/DOQI Clinical Practice Guidelines for Vascular Access: update 2000. Am J Kidney Dis. 2001;37:S137-81.

3. Vats HS. Complications of catheters: tunneled and nontunneled. Adv Chronic Kidney Dis. 2012;19:188-94.

4. Weijmer MC, Vervloet MG, ter Wee PM. Compared to tunnelled cuffed haemodialysis catheters, temporary untunnelled catheters are associated with more complications already within 2 weeks of use. Nephrol Dial Transplant. 2004;19:670-7.
5. Zhu M, Zhang W, Zhou W, Zhou Y, Fang Y, Wang Y, Zhang H, Yan Y, Ni Z, Qian J. Initial hemodialysis with a temporary catheter is associated with complications of a later permanent vascular access. Blood Purif. 2014;37:131-7.

6. Vassalotti JA, Jennings WC, Beathard GA, Neumann M, Caponi S, Fox CH, Spergel LM, Fistula First Breakthrough Initiative Community Education C. Fistula first breakthrough initiative community education C: fistula first breakthrough initiative: targeting catheter last in fistula first. Semin Dial. 2012:25:303-10.

7. Ethier J, Mendelssohn DC, Elder SJ, Hasegawa T, Akizawa T, Akiba T, Canaud BJ, Pisoni RL. Vascular access use and outcomes: an international perspective from the Dialysis Outcomes and Practice Patterns Study. Nephrol Dial Transplant. 2008;23:3219-26.

8. Centers for Medicare and Medicaid Services. End Stage Renal Disease Network Organization Program 2013 Summary Annual Report. Baltimore, MD: CMS; 2015. Available at: http://www.esrdncc.org/wpcontent/uploads/ 2014/10/SAR_2013_Report_Approved.pdf. Accessed January 22, 2015.

9. Foley RN, Chen SC, Collins AJ. Hemodialysis access at initiation in the United States, 2005 to 2007: still "catheter first". Hemodial Int. 2009;13:533-42.

10. Xue $H_{1}, \mathrm{I}$ JH, Wang W, Brunelli SM, Lazarus M, Hakim R, Lacson E, Jr. Hemodialysis access usage patterns in the incident dialysis year and associated catheter-related complications. Am J Kidney Dis. 2013;61:123-30.

11. Ethier J, Bragg-Gresham JL, Piera L, Akizawa T, Asano Y, Mason N, Gillespie BW, Young EW. Aspirin prescription and outcomes in hemodialysis patients: the Dialysis Outcomes and Practice Patterns Study (DOPPS). Am J Kidney Dis. 2007; 50:602-11.

12. Fink JC, Blahut SA, Briglia AE, Gardner JF, Light PD. Effect of center- versus patient-specific factors on variations in dialysis adequacy. J Am Soc Nephrol. 2001;12:164-9.

13. Fink JC, Hsu VD, Zhan M, Walker LD, Mullins CD, Jones-Burton C, Langenberg $P$, Seliger SL. Center effects in anemia management of dialysis patients. J Am Soc Nephrol. 2007;18:646-53.

14. Fink JC, Zhan M, Blahut SA, Soucie M, McClellan WM. Measuring the efficacy of a quality improvement program in dialysis adequacy with changes in center effects. J Am Soc Nephrol. 2002;13:2338-44.

15. Pisoni RL, Arrington CJ, Albert JM, Ethier J, Kimata N, Krishnan M, Rayner HC, Saito A, Sands JJ, Saran R, Gillespie B, Wolfe RA, Port FK. Facility hemodialysis vascular access use and mortality in countries participating in DOPPS: an instrumental variable analysis. Am J Kidney Dis. 2009:53:475-91.

16. Pisoni RL, Gillespie BW, Dickinson DM, Chen K, Kutner MH, Wolfe RA. The Dialysis Outcomes and Practice Patterns Study (DOPPS): design, data elements, and methodology. Am J Kidney Dis. 2004;44:7-15.

17. Sood MM, Manns B, Dart A, Hiebert B, Kappel J, Komenda P, Molzahn A, Naimark D, Nessim S, Rigatto C, Soroka S, Zappitelli M, Tangri N, on behalf of the Canadian Kidney Knowledge Translation and Generation Network (CANN-NET). Variation in the level of eGFR at dialysis initiation across dialysis facilities and geographic regions. Clin J Am Soc Nephrol. 2014:9:1747-56.

18. Tangri N, Moorthi R, Tighiouhart H, Meyer KB, Miskulin DC. Variation in fistula use across dialysis facilities: is it explained by case-mix? Clin J Am Soc Nephrol. 2010;5:307-13.

19. Tangri $\mathrm{N}$, Tighiouart $\mathrm{H}$, Meyer KB, Miskulin DC. Both patient and facility contribute to achieving the Centers for Medicare and Medicaid Services' pay-for-performance target for dialysis adequacy. J Am Soc Nephrol. 2011; 22:2296-302.

20. Wetmore JB, Mahnken JD, Mukhopadhyay P, Hou Q, Ellerbeck EF, Rigler SK, Spertus JA, Shireman TI. Geographic variation in cardioprotective antihypertensive medication usage in dialysis patients. Am J Kidney Dis. 2011;58:73-83.

21. Canadian Institute for Health Information: Dialysis and Renal Transplantation Canadian Institute for Health Information, Ottawa, Ontario, Canada; 2009.

22. Moist LM, Richards HA, Miskulin D, Lok CE, Yeates K, Garg AX, Trpeski L, Chapman A, Amuah J, Hemmelgarn BR. A validation study of the Canadian Organ Replacement Register. Clin J Am Soc Nephrol. 2011;6:813-8.

23. Miller LM, Vercaigne LM, Moist L, Lok CE, Tangri N, Komenda P, Rigatto C, Mojica J, Sood MM. The association between geographic proximity to a dialysis facility and use of dialysis catheters. BMC Nephrol. 2014;15:40.

24. Benner D, Hollister D, Mcallister CJ, Thiry K. The DaVita Quality Index (DQI): a measure of clinical performance. Dial Transplant. 2003;32:269-73.

25. Mendelssohn DC, Pisoni RL, Arrington CJ, Yeates KE, Leblanc M, Deziel C, Akiba T, Krishnan M, Fukuhara S, Lameire N, Port FK, Wolfe RA. A practicerelated risk score (PRS): a DOPPS-derived aggregate quality index for haemodialysis facilities. Nephrol Dial Transplant. 2008;23:3227-33. 
26. Rocco MV, Frankenfield DL, Hopson SD, McClellan WM. Relationship between clinical performance measures and outcomes among patients receiving long-term hemodialysis. Ann Intern Med. 2006;145:512-9.

27. Schaubel DE, Blake PG, Fenton SS. Effect of renal center characteristics on mortality and technique failure on peritoneal dialysis. Kidney Int. 2001:60:1517-24.

28. Schafer JL. Multiple imputation: a primer. Stat Methods Med Res. 1999;8:3-15.

29. Heck RH, Tabata LN. Multilevel and longitudinal modeling with IBM SPSS. New York: Routledge; 2010.

30. Greenland S. Principles of multilevel modelling. Int J Epidemiol. 2000;29:158-67.

31. Raudenbush SW BA. Hierarchical linear models: Applications and data analysis methods. secondth ed. Thousand Oaks, CA: Sage Publications Inc; 2002.

32. Snijders TBR. Multilevel analysis: an introduction to basic and advanced multilevel modeling. London: Sage Publications Ltd; 1990.

33. Heo M, Leon AC. Performance of a mixed effects logistic regression model for binary outcomes with unequal cluster size. J Biopharm Stat. 2005;15:513-26.

34. Rodríguez G, Elo I. Intra-class correlation in random-effects models for binary data. Stata J. 2003;3:32-46.

35. Moist LM, Trpeski L, Na Y, Lok CE. Increased hemodialysis catheter use in Canada and associated mortality risk: data from the Canadian Organ Replacement Registry 2001-2004. Clin J Am Soc Nephrol. 2008;3:1726-32.

36. Sood MM, Tangri N, Hiebert B, Kappel J, Dart A, Levin A, Manns B, Molzahn A, Naimark D, Nessim SJ, Rigatto C, Soroka SD, Zappitelli M, Komenda P, Canadian Kidney Knowledge T, Generation N. Geographic and facility-level variation in the use of peritoneal dialysis in Canada: a cohort study. CMAJ Open. 2014;2:E36-44.

37. Graham J, Hiremath S, Magner PO, Knoll GA, Burns KD. Factors influencing the prevalence of central venous catheter use in a Canadian haemodialysis centre. Nephrol Dial Transplant. 2008;23:3585-91.

38. Hughes SA, Mendelssohn JG, Tobe SW, McFarlane PA, Mendelssohn DC. Factors associated with suboptimal initiation of dialysis despite early nephrologist referral. Nephrol Dial Transplant. 2013;28:392-7.

39. Negoianu D, Berns JS. Should nephrology training programs continue to train fellows in the placement of temporary hemodialysis catheters? Semin Dial. 2014;27:245-7.

40. Hsu RK, McCulloch CE, Ku E, Dudley RA, Hsu CY. Regional variation in the incidence of dialysis-requiring AKI in the United States. Clin J Am Soc Nephrol. 2013;8:1476-81.

41. Buck J, Baker R, Cannaby AM, Nicholson S, Peters J, Warwick G. Why do patients known to renal services still undergo urgent dialysis initiation? A cross-sectional survey. Nephrol Dial Transplant. 2007;22:3240-5.

42. Crews DC, Jaar BG, Plantinga LC, Kassem HS, Fink NE, Powe NR. Inpatient hemodialysis initiation: reasons, risk factors and outcomes. Nephron Clin Pract. 2010;114:C19-28.

43. Marron B, Ortiz A, de Sequera P, Martin-Reyes G, de Arriba G, Lamas JM, Martinez Ocana JC, Arrieta J, Martinez F, Spanish Group for CKD. Impact of end-stage renal disease care in planned dialysis start and type of renal replacement therapy-a Spanish multicentre experience. Nephrol Dial Transplant. 2006;21 Suppl 2:ii51-55.

44. Mendelssohn DC, Curtis B, Yeates K, Langlois S, MacRae JM, Semeniuk LM, Suboptimal initiation of dialysis with and without early referral to a nephrologist. Nephrol Dial Transplant. 2011;26:2959-65.

\section{Submit your next manuscript to BioMed Central and we will help you at every step:}

- We accept pre-submission inquiries

- Our selector tool helps you to find the most relevant journal

- We provide round the clock customer support

- Convenient online submission

- Thorough peer review

- Inclusion in PubMed and all major indexing services

- Maximum visibility for your research

Submit your manuscript at www.biomedcentral.com/submit

) Biomed Central 